\title{
A NEW NUMERICAL TECHNIQUE FOR SOLVING SYSTEMS OF NONLINEAR FRACTIONAL PARTIAL DIFFERENTIAL EQUATIONS
}

\author{
MOUNTASSIR HAMDI CHERIF ${ }^{1 *}$ AND DJELLOUL ZIANE ${ }^{2}$
}

\begin{abstract}
In this paper, we apply an efficient method called the Aboodh decomposition method to solve systems of nonlinear fractional partial differential equations. This method is a combined form of Aboodh transform with Adomian decomposition method. The theoretical analysis of this investigated for systems of nonlinear fractional partial differential equations is calculated in the explicit form of a power series with easily computable terms. Some examples are given to shows that this method is very efficient and accurate. This method can be applied to solve others nonlinear systems problems.
\end{abstract}

\section{INTRODUCTION}

Over the last three decades, fractional calculus has been enormously developed and taken on in many fields of scientific research. One of the main reasons is its application in many scientific disciplines. The fractional calculus by its tools remains a very suitable means for the resolution of the differential systems. These generally translate mathematical or physical models of the many natural phenomena that surround us. Integro-differential equations and fractional differential systems have recently proved to be very useful in the field of physics, engineering, control processing for visco-elastic systems, diffusion, .... We find that many researchers have been interested in solving this kind of linear and nonlinear differential equations also systems of fractional differential equations. Consequently, the investigation of the exact solutions to nonlinear equations play an important role in the study of nonlinear physical phenomena, although the nonlinear differential equations are the most complex in the solution compared with linear differential equations.

Integral transformations such as Laplace, Sumudu, natural, Elzaki and Aboodh are unable to solve the nonlinear differential equations. So, we find some researchers are working on the combined of these transformations with many methods, among them we find the Adomian decomposition method. This method was introduced in the 1980s by George Adomian (1923-1996), and it was applied to many problems ( [1]- [4]). The Adomian decomposition method was coupled with Laplace transform method [14], with Sumudu transform method [9], with Elzaki transform method [10], with natural transform method [11] and with Aboodh transform method. Aboodh transform is derived from the classical Fourier integral. Based on the mathematical simplicity of the Aboodh transform and its fundamental properties, Aboodh transform was introduced by Khalid Aboodh in 2013, to facilitate the process of solving ordinary and partial differential equations in the time domain. This transformation has deeper connection with the Laplace and Elzaki transform [8]. The coupling of Adomian decomposition method with Aboodh transform method has been applied for solving linear and nonlinear equations [17], to solve system of linear and nonlinear partial differential equations [13] and for solving time-fractional

diffusion equation [16].

The objective of this study is coupling the Adomian decompositionmethod (ADM) with Aboodh transform in the sense of fractional derivative, then we apply this modified method to solve some examples related with systems of nolinear fractional partial differential equations.

Received $21^{\text {st }}$ July, 2017; accepted $19^{\text {th }}$ October, 2017; published $1^{\text {st }}$ November, 2017.

2010 Mathematics Subject Classification. 26A33, 44A05, 34K37, 35F61.

Key words and phrases. Aboodh transform; Caputo fractional derivative; Adomain decomposition method; system of nonlinear fractional differential equations.

(C) 2017 Authors retain the copyrights of their papers, and all open access articles are distributed under the terms of the Creative Commons Attribution License. 


\section{BASIC DEFINITIONS}

In this section, we give some basic notions about fractional calculus, Aboodh transform and Aboudh transform of fractional derivatives which are used further in this paper.

2.1. Fractional calculus. We give some basic definitions and properties of the fractional calculus theory as the Riemann-Liouville fractional integrals and Caputo fractional derivative (see [6], [7]).

Definition 2.1. Let $\Omega=[a, b](-\infty<a<b<+\infty)$ be a finite interval on the real axis $\mathbb{R}$. The Riemann-Liouville fractional integral $I_{0+}^{\alpha} f$ of order $\alpha \in \mathbb{R}(\alpha>0)$ is defined by

$$
\begin{aligned}
\left(I_{0+}^{\alpha} f\right)(t) & =\frac{1}{\Gamma(\alpha)} \int_{0}^{t} \frac{f(\tau) d \tau}{(t-\tau)^{1-\alpha}}, \quad t>0, \alpha>0 \\
\left(I_{0+}^{0} f\right)(t) & =f(t)
\end{aligned}
$$

Here $\Gamma(\cdot)$ is the gamma function.

Theorem 2.1. Let $\alpha \geqslant 0$ and let $n=[\alpha]+1$. If $f(t) \in A C^{n}[a, b]$, then the Caputo fractional derivative $\left({ }^{c} D_{0+}^{\alpha} f\right)(t)$ exist almost evrywhere on $[a, b]$. If $\alpha \notin \mathbb{N},\left({ }^{c} D_{0^{+}}^{\alpha} f\right)(t)$ is represented by

$$
\left({ }^{c} D_{0+}^{\alpha} f\right)(t)=\frac{1}{\Gamma(n-\alpha)} \int_{0}^{t} \frac{f^{(n)}(\tau) d \tau}{(t-\tau)^{\alpha-n+1}}
$$

where $D=\frac{d}{d x}$ and $n=[\alpha]+1$.

Remark 2.1. In this paper, we consider the time-fractional derivative in the Caputo's sense. When $\alpha \in \mathbb{R}^{+}$, the time-fractional derivative is defined as

$$
\begin{aligned}
\left({ }^{c} D_{t}^{\alpha} u\right)(x, t) & =\frac{\partial^{\alpha} u(x, t)}{\partial t^{\alpha}} \\
& =\left\{\begin{array}{r}
\frac{1}{\Gamma(m-\alpha)} \int_{0}^{t}(t-\tau)^{m-\alpha-1} \frac{\partial^{m} u(x, \tau)}{\partial \tau^{m}}, \quad m-1<\alpha<m, \\
\frac{\partial^{m} u(x, t)}{\partial t^{m}}, \quad \alpha=m,
\end{array}\right.
\end{aligned}
$$

where $m \in \mathbb{N}^{*}$.

2.2. Definitions and properties of the Aboodh transform. The Aboodh transform was defined by K. S. Aboodh [12] in 2013. In this section, we give some basic definitions and properties of this transform (see [12], [15], [16]).

The Aboodh transform is defined for functions of exponential order. We consider functions belonging to a class $B$, where $B$ defined by

$$
B=\left\{u(t):|u(t)|<M e^{k_{j}|t|}, \text { if } t \in(-1)^{j} \times\left[0, \infty, j=1,2 ; M, k_{1}, k_{2}>0\right\} .\right.
$$

Definition 2.2. The Aboodh integral transform of the function $u$ in $B$ is defined by the integral equation

$$
A[u(t)]=U(v)=\frac{1}{v} \int_{0}^{\infty} u(t) e^{-v t} d t ; \quad t \geq o, \quad v \in\left(k_{1}, k_{2}\right) .
$$

The variable $v$ in this transform is used to factor the variable $t$ in the argument of the function $u$.

Proposition 2.1. The Aboodh transform of the time-fractional derivative in the Caputo's sense is defined as

$$
A\left[\left({ }^{c} D_{0+}^{\alpha} u\right)(t) ; v\right]=v^{\alpha} A[u(t)]-\sum_{k=0}^{n-1} \frac{u^{(k)}(0)}{v^{2-\alpha+k}}, \quad n-1<\alpha \leq n, \quad n=1,2, \ldots
$$

And the Aboodh transform of the function $u(x, t)$ with Caputo fractional derivative of order $\alpha$ is given by 


$$
A\left[\left({ }^{c} D_{0+}^{\alpha} u\right)(x, t) ; v\right]=v^{\alpha} A[u(x, t)]-\sum_{k=0}^{n-1} \frac{u^{(k)}(x, 0)}{v^{2-\alpha+k}}, \quad n-1<\alpha \leq n, \quad n=1,2, \ldots
$$

2.2.1. Somme properties of the Aboodh transform

. 1. The Aboodh transform of the $n t h$ derivative of $u(t)$ is given by

$$
A\left[u^{(n)}(t)\right]=U_{n}(v)=v^{n} A[u(t)]-\sum_{k=0}^{n-1} \frac{u^{(k)}(0)}{v^{2-n+k}}
$$

2. Some elementary functions and their transformations

\begin{tabular}{|l|l|}
\hline$u(t)$ & $A[u(t)]$ \\
\hline 1 & $\frac{1}{v^{2}}$ \\
\hline$t$ & $\frac{1}{v^{3}}$ \\
\hline$t^{n}$ & $\frac{n !}{v^{n+2}}, \quad n=0,1,2, \ldots$ \\
\hline$t^{\alpha}$ & $\frac{\Gamma(\alpha+1)}{v^{\alpha+2}}, \alpha \geqslant 0$. \\
\hline
\end{tabular}

\section{Analysis of fractional Aboodh decomposition method}

To illustrate the basic idea of this method, we consider the general system of nonlinear fractional partial differential equations of the form

$$
\begin{aligned}
& { }^{c} D_{t}^{\alpha} u(x, t)+R w(x, t)+N u(x, t)=h_{1}(x, t), \\
& { }^{c} D_{t}^{\beta} w(x, t)+R u(x, t)+N w(x, t)=h_{2}(x, t),
\end{aligned}
$$

where $n-1<\alpha, \beta \leqslant n, n=1,2, \ldots$ and the initial conditions

$$
\begin{aligned}
& {\left[\frac{\partial^{n-1} u(x, t)}{\partial t^{n-1}}\right]_{t=0}=f_{n-1}(x), \quad n=1,2, \ldots} \\
& {\left[\frac{\partial^{n-1} w(x, t)}{\partial t^{n-1}}\right]_{t=0}=g_{n-1}(x), \quad n=1,2, \ldots}
\end{aligned}
$$

${ }^{c} D_{t}^{\alpha} u(x, t),{ }^{c} D_{t}^{\beta} w(x, t)$ are the Caputo fractional derivatives of the functions $u(x, t), w(x, t)$ respectively, $R$ is the linear differential operator, $N$ represent the general nonlinear differential operator, and $h_{1}(x, t), h_{2}(x, t)$ are the source terms.

Applying the Aboodh transform on both sides of (3.1) and using the differentiation property of this transform (2.3), we obtain

$$
\begin{aligned}
& A[u(x, t)]=\frac{1}{v^{\alpha}} \sum_{k=0}^{n-1} \frac{u^{(k)}(x, 0)}{v^{2-\alpha+k}}+\frac{1}{v^{\alpha}} A\left[h_{1}(x, t)\right]-\frac{1}{v^{\alpha}} A[R w(x, t)+N u(x, t)], \\
& A[w(x, t)]=\frac{1}{v^{\beta}} \sum_{k=0}^{n-1} \frac{w^{(k)}(x, 0)}{v^{2-\beta+k}}+\frac{1}{v^{\beta}} A\left[h_{2}(x, t)\right]-\frac{1}{v^{\beta}} A[R u(x, t)+N w(x, t)] .
\end{aligned}
$$

Taking the inverse Aboodh transform on both sides of equations in system (3.3) and then by using initial conditions (3.2), we have

$$
\begin{aligned}
v(x, t) & =G(x, t)-A^{-1}\left(\frac{1}{v^{\alpha}} A[R w(x, t)+N u(x, t)]\right), \\
w(x, t) & =H(x, t)-A^{-1}\left(\frac{1}{v^{\beta}} A[R u(x, t)+N w(x, t)]\right),
\end{aligned}
$$

where $G(x, t)$ and $H(x, t)$ are represents the terms arising from the nonhomogeneous terms and the prescribed initial conditions. Now, we represent solutions as the following infinite series

$$
u(x, t)=\sum_{n=0}^{\infty} u_{n}(x, t), w(x, t)=\sum_{m=0}^{\infty} w_{n}(x, t)
$$

and the nonlinear terms can be decomposed as

$$
N u(x, t)=\sum_{n=0}^{\infty} C_{n}, \quad N w(x, t)=\sum_{n=0}^{\infty} D_{n}
$$

where $C_{n}$ and $D_{n}$ are Adomian polynomials [5], and they can be calculated by the formulas given below 


$$
C_{n}=\frac{1}{n !} \frac{\partial^{n}}{\partial \lambda^{n}}\left[N\left(\sum_{i=0}^{\infty} \lambda^{i} u_{i}\right)\right]_{\lambda=0}, \quad D_{n}=\frac{1}{n !} \frac{\partial^{n}}{\partial \lambda^{n}}\left[N\left(\sum_{i=0}^{\infty} \lambda^{i} w_{i}\right)\right]_{\lambda=0}, i=0,1,2, \cdots
$$

Using (3.5) and (3.6), we can rewrite (3.4) as

$$
\begin{aligned}
\sum_{n=0}^{\infty} u_{n}(x, t) & =G(x, t)-A^{-1}\left[\frac{1}{v^{\alpha}} A\left[R \sum_{n=0}^{\infty} w_{n}+\sum_{n=0}^{\infty} C_{n}\right]\right] \\
\sum_{n=0}^{\infty} w_{n}(x, t) & =H(x, t)-A^{-1}\left[\frac{1}{v^{\beta}} A\left[R \sum_{n=0}^{\infty} u_{n}+\sum_{n=0}^{\infty} D_{n}\right]\right]
\end{aligned} .
$$

By comparing both sides of (3.8), we get

$$
\begin{gathered}
u_{0}(x, t)=G(x, t), \\
u_{1}(x, t)=-A^{-1}\left[\frac{1}{v^{\alpha}} A\left[R w_{0}(x, t)+C_{0}\right]\right], \\
u_{2}(x, t)=-A^{-1}\left[\frac{1}{v^{\alpha}} A\left[R w_{1}(x, t)+C_{1}\right]\right] .
\end{gathered}
$$

and

$$
\begin{gathered}
w_{0}(x, t)=H(x, t), \\
w_{1}(x, t)=-A^{-1}\left[\frac{1}{v^{\beta}} A\left[R u_{0}(x, t)+D_{0}\right]\right], \\
w_{2}(x, t)=-A^{-1}\left[\frac{1}{v^{\beta}} A\left[R u_{1}(x, t)+D_{1}\right]\right], \\
\vdots
\end{gathered}
$$

We continue in this manner to obtain the general recursive relations

$$
\begin{array}{ll}
u_{n+1}(x, t)=-A^{-1}\left[\frac{1}{v^{\alpha}} A\left[R w_{n}(x, t)+C_{n}\right]\right], & n \geqslant 1 \\
w_{n+1}(x, t)=-A^{-1}\left[\frac{1}{v^{\beta}} A\left[R u_{n}(x, t)+D_{n}\right]\right], & n \geqslant 1
\end{array} .
$$

Finally, the approximate solution is calculated by

$$
u(x, t)=\lim _{N \rightarrow \infty} \sum_{n=0}^{N} u_{n}(x, t) \quad w(x, t)=\lim _{N \rightarrow \infty} \sum_{n=0}^{N} w_{n}(x, t) .
$$

\section{Applications}

In this section, we apply the Aboodh decomposition transform method for solving systems of nonlinear fractional partial differential equations.

Example 4.1. Consider the system of nonlinear partial differential equations with time-fractional derivatives

$$
\begin{array}{ll}
{ }^{c} D_{t}^{\alpha} u(x, t)+w(x, t) u_{x}(x, t)+u(x, t)=1, & 0 \leq \alpha<1 \\
{ }^{c} D_{t}^{\beta} w(x, t)-u(x, t) w_{x}(x, t)-w(x, t)=1, & 0 \leq \beta<1
\end{array}
$$

with the initial conditions

$$
\begin{gathered}
u(x, 0)=e^{x} \\
w(x, 0)=e^{-x}
\end{gathered}
$$

For $\alpha=\beta=1$, the exact solution of (4.1) is given by

$$
\begin{aligned}
& u(x, t)=e^{x-t} \\
& w(x, t)=e^{t-x}
\end{aligned}
$$

Applying the Aboodh transform on both sides of (4.1) and using its differentiation property, we get

$$
\begin{gathered}
A[u(x, t)]=\frac{e^{x}}{v^{2}}+\frac{1}{v^{\alpha}} A\left[1-w(x, t) u_{x}(x, t)-u(x, t)\right], \\
A[w(x, t)]=\frac{e^{-x}}{v^{2}}+\frac{1}{v^{\beta}} A\left[1+u(x, t) w_{x}(x, t)+w(x, t)\right] .
\end{gathered}
$$

Taking the inverse Aboodh transform on both sides of (4.4), we obtain 


$$
\begin{gathered}
u(x, t)=e^{x}+\frac{t^{\alpha}}{\Gamma(\alpha+1)}-A^{-1}\left(\frac{1}{v^{\alpha}} A\left[w(x, t) u_{x}(x, t)+u(x, t)\right]\right), \\
w(x, t)=e^{-x}+\frac{t^{\beta}}{\Gamma(\beta+1)}+A^{-1}\left(\frac{1}{v^{\beta}} A\left[u(x, t) w_{x}(x, t)+w(x, t)\right]\right) .
\end{gathered}
$$

We represent the approximate solution as the following infinite series

$$
u(x, t)=\sum_{n=0}^{\infty} u_{n}(x, t), \quad w(x, t)=\sum_{m=0}^{\infty} w_{n}(x, t)
$$

Note that these nonlinear terms

$$
w u_{x}=\sum_{n=0}^{\infty} C_{n}, \quad u w_{x}=\sum_{n=0}^{\infty} D_{n}
$$

are the Adomian polynomials (see [5]). The first few components of $C_{n}$ and $D_{n}$ polynomials are given by

$$
\begin{gathered}
C_{0}=w_{0} u_{0 x} \\
C_{1}=w_{0} u_{1 x}+w_{1} u_{0 x} \\
C_{2}=w_{0} u_{2 x}+w_{2} u_{0 x}+w_{1} u_{1 x}
\end{gathered}
$$

And

$$
\begin{gathered}
D_{0}=u_{0} w_{0 x} \\
D_{1}=u_{0} w_{1 x}+u_{1} w_{0 x} \\
D_{2}=u_{0} w_{2 x}+u_{2} w_{0 x}+u_{1} w_{1 x}
\end{gathered}
$$

Substituting (4.6) and (4.7) in (4.5), we have

$$
\begin{gathered}
\sum_{n=0}^{\infty} u_{n}(x, t)=e^{x}+\frac{t^{\alpha}}{\Gamma(\alpha+1)}-A^{-1}\left(\frac{1}{v^{\alpha}} A\left[\sum_{n=0}^{\infty} C_{n}+\sum_{n=0}^{\infty} u_{n}(x, t)\right]\right) \\
\sum_{n=0}^{\infty} w_{n}(x, t)=e^{-x}+\frac{t^{\beta}}{\Gamma(\beta+1)}+A^{-1}\left(\frac{1}{v^{\beta}} A\left[\sum_{n=0}^{\infty} D_{n}+\sum_{m=0}^{\infty} w_{n}(x, t)\right]\right)
\end{gathered} .
$$

By comparing both sides of (4.8), we can easily generate the recursive relations

$$
\begin{gathered}
u_{0}(x, t)=e^{x}+\frac{t^{\alpha}}{\Gamma(\alpha+1)} \\
u_{1}(x, t)=-A^{-1}\left(\frac{1}{v^{\alpha}} A\left[C_{0}+u_{0}(x, t)\right]\right) \\
u_{2}(x, t)=-A^{-1}\left(\frac{1}{v^{\alpha}} A\left[C_{1}+u_{1}(x, t)\right]\right) \\
u_{3}(x, t)=-A^{-1}\left(\frac{1}{v^{\alpha}} A\left[C_{2}+u_{2}(x, t)\right]\right) \\
\vdots \\
u_{n+1}(x, t)=-A^{-1}\left(\frac{1}{v^{\alpha}} A\left[C_{n}+u_{n}(x, t)\right]\right), \quad n \geq 0 .
\end{gathered}
$$

And

$$
\begin{gathered}
w_{0}(x, t)=e^{-x}+\frac{t^{\beta}}{\Gamma(\beta+1)} \\
w_{1}(x, t)=A^{-1}\left(\frac{1}{v^{\beta}} A\left[D_{0}+w_{0}(x, t)\right]\right) \\
w_{2}(x, t)=A^{-1}\left(\frac{1}{v^{\beta}} A\left[D_{1}+w_{1}(x, t)\right]\right) \\
\left.w_{3}(x, t)=A^{-1} \frac{1}{v^{\beta}} A\left[D_{2}+w_{2}(x, t)\right]\right) \\
\vdots \\
w_{n+1}(x, t)=A^{-1}\left(\frac{1}{v^{\beta}} A\left[D_{n}+w_{n}(x, t)\right]\right), \quad n \geq 0 .
\end{gathered}
$$

The first few components of the unknown functions $u_{n}(x, t)$ and $w_{n}(x, t)$ are given as follows 


$$
\begin{aligned}
& u_{1}(x, t)=-\frac{1+e^{x}}{\Gamma(\alpha+1)} t^{\alpha}-\frac{e^{x}}{\Gamma(\alpha+\beta+1)} t^{\alpha+\beta}-\frac{t^{2 \alpha}}{\Gamma(2 \alpha+1)} \\
& w_{1}(x, t)=\frac{-1+e^{-x}}{\Gamma(\beta+1)} t^{\beta}-\frac{e^{-x}}{\Gamma(\alpha+\beta+1)} t^{\alpha+\beta}+\frac{t^{2 \beta}}{\Gamma(2 \beta+1)}
\end{aligned} .
$$

And

$$
\begin{aligned}
u_{2}(x, t)= & \frac{2+e^{x}}{\Gamma(2 \alpha+1)} t^{2 \alpha}+\frac{e^{x}-1}{\Gamma(\alpha+\beta+1)} t^{\alpha+\beta}+\left(1+2 e^{x}+\frac{\Gamma(\alpha+\beta+1) e^{x}}{\Gamma(\alpha+1) \Gamma(\beta+1)}\right) \frac{t^{2 \alpha+\beta}}{\Gamma(2 \alpha+\beta+1)}+ \\
& \frac{\Gamma(\alpha+2 \beta+1) e^{x}}{\Gamma(\beta+1) \Gamma(\alpha+\beta+1) \Gamma(2 \alpha+2 \beta+1)} t^{2 \alpha+2 \beta}-\frac{e^{x}}{\Gamma(\alpha+2 \beta+1)} t^{\alpha+2 \beta}+\frac{t^{3 \alpha}}{\Gamma(3 \alpha+1)} \\
w_{2}(x, t)= & \frac{-2+e^{-x}}{\Gamma(2 \beta+1)} t^{2 \beta}+\frac{1+e^{-x}}{\Gamma(\alpha+\beta+1)} t^{\alpha+\beta}+\left(2-e^{-x}+\frac{\Gamma(\alpha+\beta+1) e^{-x}}{\Gamma(\alpha+1) \Gamma(\beta+1)}\right) \frac{t^{\alpha+2 \beta}}{\Gamma(\alpha+2 \beta+1)}+ \\
& \frac{\Gamma(2 \alpha+\beta+1) e^{-x}}{\Gamma(\alpha+1) \Gamma(\alpha+\beta+1) \Gamma(2 \alpha+2 \beta+1)} t^{2 \alpha+2 \beta}+\frac{e^{-x}}{\Gamma(2 \alpha+\beta+1)} t^{2 \alpha+\beta}+\frac{t^{3 \beta}}{\Gamma(3 \beta+1)}
\end{aligned}
$$

In the same manner, we can find the other components.

Finally, the series solution of the unknown functions $u(x, t)$ and $w(x, t)$ of (4.1) are given by

$$
\begin{aligned}
u(x, t)= & e^{x}-\frac{e^{x}}{\Gamma(\alpha+1)} t^{\alpha}+\frac{1+e^{x}}{\Gamma(2 \alpha+1)} t^{2 \alpha}-\frac{1}{\Gamma(\alpha+\beta+1)} t^{\alpha+\beta}+ \\
& \left(1+2 e^{x}+\frac{\Gamma(\alpha+\beta+1) e^{x}}{\Gamma(\alpha+1) \Gamma(\beta+1)}\right) \frac{t^{2 \alpha+\beta}}{\Gamma(2 \alpha+\beta+1)}+ \\
& \frac{\Gamma(\alpha+2 \beta+1) e^{x}}{\Gamma(\beta+1) \Gamma(\alpha+\beta+1) \Gamma(2 \alpha+2 \beta+1)} t^{2 \alpha+2 \beta}-\frac{e^{x}}{\Gamma(\alpha+2 \beta+1)} t^{\alpha+2 \beta}+\frac{t^{3 \alpha}}{\Gamma(3 \alpha+1)}+\ldots \\
w(x, t)= & e^{-x}+\frac{e^{-x}}{\Gamma(\beta+1)} t^{\beta}+\frac{-1+e^{-x}}{\Gamma(2 \beta+1)} t^{2 \beta}+\frac{e^{-x}}{\Gamma(\alpha+\beta+1)} t^{\alpha+\beta}+ \\
& \left(2-e^{-x}+\frac{\Gamma(\alpha+\beta+1) e^{-x}}{\Gamma(\alpha+1) \Gamma(\beta+1)}\right) \frac{t^{\alpha+2 \beta}}{\Gamma(\alpha+2 \beta+1)}+ \\
& \frac{\Gamma(2 \alpha+\beta+1) e^{-x}}{\Gamma(\alpha+1) \Gamma(\alpha+\beta+1) \Gamma(2 \alpha+2 \beta+1)} t^{2 \alpha+2 \beta}+\frac{e^{-x}}{\Gamma(2 \alpha+\beta+1)} t^{2 \alpha+\beta}+\frac{t^{3 \beta}}{\Gamma(3 \beta+1)}+\ldots
\end{aligned}
$$

When $\alpha=1$ and $\beta=1$, the series solutions of (4.1) are

$$
\begin{gathered}
u(x, t)=u_{0}(x, t)+u_{1}(x, t)+u_{2}(x, t)+\ldots=e^{x}\left(1-t+\frac{t^{2}}{2 !}-\frac{t^{3}}{3 !}+\ldots\right)=e^{x-t} \\
w(x, t)=w_{0}(x, t)+w_{1}(x, t)+w_{2}(x, t)+\ldots=e^{-x}\left(1+t+\frac{t^{2}}{2 !}+\frac{t^{3}}{3 !}+\ldots\right)=e^{-x+t}
\end{gathered}
$$

which is the exact solution of nonlinear system given in (4.3). 
Example 4.2. We consider the following system of nonlinear partial differential equations with timefractional derivatives

$$
\begin{gathered}
{ }^{c} D_{t}^{\alpha} u=-u-h_{x} w_{y}+h_{y} w_{x}, \quad 0 \leq \alpha<1 \\
{ }^{c} D_{t}^{\beta} h=h, \quad 0 \leq \beta<1 \\
{ }^{c} D_{t}^{\gamma} w=w-u_{x} w_{x}-u_{y} w_{y}, \quad 0 \leq \gamma<1
\end{gathered}
$$

with the initial conditions

$$
u(x, y, 0)=x+y ; \quad h(x, y, 0)=1+x-y ; \quad w(x, y, 0)=-x+y
$$

Taking Aboodh transform with its differentiation property of (4.11) subject to the initial conditions (4.12), we have

$$
\begin{gathered}
A[u]=\frac{x+y}{v^{2}}+\frac{1}{v^{\alpha}} A\left[-u-h_{x} w_{y}+h_{y} w_{x}\right] \\
A[h]=\frac{1+x-y}{v^{2}}+\frac{1}{v^{\beta}} A[h] \\
A[w]=\frac{-x+y}{v^{2}}+\frac{1}{v^{\gamma}} A\left[w-u_{x} w_{x}-u_{y} w_{y}\right]
\end{gathered}
$$

Now, we apply the inverse Aboodh transform on both sides of (4.13), we obtain

$$
\begin{gathered}
u(x, y, t)=x+y+A^{-1}\left(\frac{1}{v^{\alpha}} A\left[-u-h_{x} w_{y}+h_{y} w_{x}\right]\right) \\
h(x, y, t)=1+x-y+A^{-1}\left(\frac{1}{v^{\beta}} A[h]\right) \\
w(x, y, t)=-x+y+A^{-1}\left(\frac{1}{v^{\gamma}} A\left[w-u_{x} w_{x}-u_{y} w_{y}\right]\right)
\end{gathered}
$$

We represent solutions as the following infinite series

$$
u(x, y, t)=\sum_{n=0}^{\infty} u_{n}(x, y, t), h(x, y, t)=\sum_{n=0}^{\infty} h_{n}(x, y, t), w(x, y, t)=\sum_{n=0}^{\infty} w_{n}(x, y, t) .
$$

Note that these nonlinear terms

$$
h_{x} w_{y}=\sum_{n=0}^{\infty} A_{n} ; h_{y} w_{x}=\sum_{m=0}^{\infty} B_{n} ; u_{x} w_{x}=\sum_{n=0}^{\infty} C_{n} ; u_{y} w_{y}=\sum_{m=0}^{\infty} D_{n}
$$

are the Adomian polynomials [5].

Substituting (4.15) and (4.16) in (4.14), we have

$$
\begin{gathered}
\sum_{n=0}^{\infty} u_{n}(x, y, t)=x+y+A^{-1}\left(\frac{1}{v^{\alpha}} A\left[-\sum_{n=0}^{\infty} u_{n}-\sum_{n=0}^{\infty} A_{n}+\sum_{m=0}^{\infty} B_{n}\right]\right), \\
\sum_{m=0}^{\infty} h_{n}(x, y, t)=1+x-y+A^{-1}\left(\frac{1}{v^{\beta}} A\left[\sum_{n=0}^{\infty} h_{n}\right]\right), \\
\sum_{n=0}^{\infty} w_{n}(x, y, t)=-x+y+A^{-1}\left(\frac{1}{v^{\gamma}} A\left[\sum_{n=0}^{\infty} w_{n}-\sum_{m=0}^{\infty} C_{n}-\sum_{n=0}^{\infty} D_{n}\right]\right) .
\end{gathered}
$$

By comparing both sides of equations (4.17), we can easily generate the recursive relations

$$
\begin{gathered}
u_{0}(x, y, t)=x+y \\
u_{1}(x, y, t)=A^{-1}\left(\frac{1}{v^{\alpha}} A\left[-u_{0}-A_{0}+B_{0}\right]\right) \\
u_{2}(x, y, t)=A^{-1}\left(\frac{1}{v^{\alpha}} A\left[-u_{1}-A_{1}+B_{1}\right]\right) \\
u_{3}(x, y, t)=A^{-1}\left(\frac{1}{v^{\alpha}} A\left[-u_{2}-A_{2}+B_{2}\right]\right) \\
\vdots \\
u_{n+1}(x, y, t)=A^{-1}\left(\frac{1}{v^{\alpha}} A\left[-u_{n}-A_{n}+B_{n}\right]\right), \quad n \geq 0 .
\end{gathered}
$$

And 


$$
\begin{gathered}
h_{0}(x, y, t)=1+x-y \\
h_{1}(x, y, t)=A^{-1}\left(\frac{1}{v^{\beta}} A\left[h_{0}\right]\right) \\
h_{2}(x, y, t)=A^{-1}\left(\frac{1}{v^{\beta}} A\left[h_{1}\right]\right) \\
h_{3}(x, y, t)=A^{-1}\left(\frac{1}{v^{\beta}} A\left[h_{2}\right]\right) \\
\vdots \\
h_{n+1}(x, y, t)=A^{-1}\left(\frac{1}{v^{\beta}} A\left[h_{n}\right]\right), \quad n \geq 0 .
\end{gathered}
$$

Finally

$$
\begin{gathered}
w_{0}(x, y, t)=-x+y \\
w_{1}(x, y, t)=A^{-1}\left(\frac{1}{v^{\gamma}} A\left[w_{0}-C_{0}-D_{0}\right]\right) \\
w_{2}(x, y, t)=A^{-1}\left(\frac{1}{v^{\gamma}} A\left[w_{1}-C_{1}-D_{1}\right]\right) \\
\left.w_{3}(x, y, t)=A^{-1} \frac{1}{\frac{1}{v^{\gamma}}} A\left[w_{2}-C_{2}-D_{2}\right]\right) \\
\vdots \\
w_{n+1}(x, y, t)=A^{-1}\left(\frac{1}{v^{\gamma}} A\left[w_{n}-C_{n}-D_{n}\right]\right), \quad n \geq 0 .
\end{gathered}
$$

The first few components of the unknown functions $u_{n}(x, y, t), h_{n}(x, y, t)$ and $w_{n}(x, y, t)$ are given as follows

$$
\begin{gathered}
u_{1}(x, y, t)=A^{-1}\left(\frac{1}{v^{\alpha}} A\left[-u_{0}-h_{0 x} w_{0 y}+h_{0 y} w_{0 x}\right]\right) \\
=-(x+y) \frac{t^{\alpha}}{\Gamma(\alpha+1)} \\
h_{1}(x, y, t)=A^{-1}\left(\frac{1}{v^{\beta}} A\left[h_{0}\right]\right) \\
=(1+x-y) \frac{t^{\beta}}{\Gamma(\beta+1)} \\
w_{1}(x, y, t)=A^{-1}\left(\frac{1}{v^{\gamma}} A\left[w_{0}-u_{0 x} w_{0 x}-u_{0 y} w_{0 y}\right]\right) \\
=(-x+y) \frac{t^{\lambda}}{\Gamma(\lambda+1)}
\end{gathered}
$$

The second component of each solution series is given by the following formulas

$$
\begin{gathered}
u_{2}(x, y, t)=A^{-1}\left(\frac{1}{v^{\alpha}} A\left[-u_{1}-h_{0 x} w_{1 y}-h_{1 x} w_{0 y}+h_{0 y} w_{1 x}+h_{1 y} w_{0 x}\right]\right) \\
=(x+y) \frac{t^{2 \alpha}}{\Gamma(2 \alpha+1)} \\
h_{2}(x, y, t)=A^{-1}\left(\frac{1}{v^{\beta}} A\left[h_{1}\right]\right) \\
=(1+x-y) \frac{t^{2 \beta}}{\Gamma(2 \beta+1)} \\
w_{2}(x, y, t)=A^{-1}\left(\frac{1}{v^{\gamma}} A\left[w_{1}-u_{0 x} w_{1 x}-u_{1 x} w_{0 x}-u_{0 y} w_{1 y}-u_{1 y} w_{0 y}\right]\right) \\
=(-x+y) \frac{t^{2 \lambda}}{\Gamma(2 \lambda+1)}
\end{gathered}
$$

We continue the calculations to find

$$
\begin{gathered}
u_{3}(x, y, t)=A^{-1}\left(\frac{1}{v^{\alpha}} A\left[-u_{2}-A_{2}+B_{2}\right]\right) \\
=-(x+y) \frac{t^{2 \alpha}}{\Gamma(2 \alpha+1)} \\
\vdots \\
u_{n}(x, y, t)=(-1)^{n}(x+y) \frac{t^{n \alpha}}{\Gamma(n \alpha+1)} . \\
h_{3}(x, y, t)=A^{-1}\left(\frac{1}{v^{\beta}} A\left[h_{2}\right]\right) \\
=(1+x-y) \frac{t^{3 \beta}}{\Gamma(3 \beta+1)} \\
\vdots \\
h_{n}(x, y, t)=(1+x-y) \frac{t^{n \beta}}{\Gamma(n \beta+1)} .
\end{gathered}
$$




$$
\begin{gathered}
w_{3}(x, y, t)=A^{-1}\left(\frac{1}{v^{\gamma}} A\left[w_{2}-C_{2}-D_{2}\right]\right) \\
=(-x+y) \frac{t^{3 \lambda}}{\Gamma(3 \lambda+1)} \\
\vdots \\
w_{n}(x, y, t)=(-x+y) \frac{t^{n \lambda}}{\Gamma(n \lambda+1)} .
\end{gathered}
$$

Finally, the series solutions of the system (4.11) are given by

$$
\begin{gathered}
u(x, y, t)=\sum_{n=0}^{\infty} u_{n}(x, y, t) \\
=(x+y)\left(1-\frac{t^{\alpha}}{\Gamma(\alpha+1)}+\frac{t^{2 \alpha}}{\Gamma(2 \alpha+1)}-\frac{t^{3 \alpha}}{\Gamma(3 \alpha+1)}+\ldots \pm \frac{t^{n \alpha}}{\Gamma(n \alpha+1)} \pm \ldots\right) \\
=(x+y) \sum_{n=0}^{\infty} \frac{\left(-t^{\alpha}\right)^{n}}{\Gamma(n \alpha+1)}=(x+y) E_{\alpha}\left(-t^{\alpha}\right) . \\
h(x, y, t)=\sum_{n=0}^{\infty} h_{n}(x, y, t) \\
=(1+x-y)\left(1+\frac{t^{\beta}}{\Gamma(\beta+1)}+\frac{t^{2 \beta}}{\Gamma(2 \beta+1)}+\frac{t^{3 \beta}}{\Gamma(3 \beta+1)}+\ldots+\frac{t^{n \beta}}{\Gamma(n \beta+1)}+\ldots\right) \\
=(1+x-y) \sum_{n=0}^{\infty} \frac{\left(t^{\beta}\right)^{n}}{\Gamma(n \beta+1)}=(1+x-y) E_{\beta}\left(t^{\beta}\right) . \\
w(x, y, t)=\sum_{n=0}^{\infty} w_{n}(x, y, t) \\
=(-x+y)\left(1+\frac{t^{\lambda}}{\Gamma(\lambda+1)}+\frac{\left(t^{\lambda}\right)^{2}}{\Gamma(2 \lambda+1)}+\frac{\left(t^{\lambda}\right)^{3}}{\Gamma(3 \lambda+1)}+\ldots+\frac{\left(t^{\lambda}\right)^{n}}{\Gamma(n \lambda+1)}+\ldots\right) \\
=(-x+y) \sum_{n=0}^{\infty} \frac{\left(t^{\lambda}\right)^{n}}{\Gamma(n \lambda+1)}=(-x+y) E_{\lambda}\left(t^{\lambda}\right) .
\end{gathered}
$$

Where, $E_{\alpha}, E_{\beta}$ and $E_{\lambda}$ are the Mittag-Leffler functions.

When $\alpha=1, \beta=1$ and $\lambda=1$, we get

$$
\begin{aligned}
u(x, y, t)=(x+y) E_{\alpha}(-t) & =(x+y) e^{-t} . \\
h(x, y, t)=(1+x-y) E_{\beta}(t) & =(1+x-y) e^{t} . \\
w(x, y, t)=(-x+y) E_{\lambda}(t) & =(-x+y) e^{t} .
\end{aligned}
$$

These are the exact solutions of nonlinear system (4.11).

\section{Conclusion}

In this work, the Aboodh transform method combined with Adomian decomposition method has been successfully applied to solve systems of nonliner fractional partial differential equations. The approximate solutions obtained by this method are compared with the exact solutions. Thus, the results show that this method is a powerful mathematical tool for solving systems of nonlinear fractional partial differential equations in other areas of science.

\section{REFERENCES}

[1] G. Adomian, Nonlinear Stochastic Systems Theory and Applications to Physics, Kluwer Academic Publishers, Netherlands, (1989).

[2] G. Adomian and R. Rach, Equality of partial solutions in the decomposition method for linear or nonlinear partial differential equations, Comput. Math. Appl., 10 (1990), 9-12.

[3] G. Adomian, Solving Frontier Problems of Physics: The Decomposition Method, Kluwer Academic Publishers, Boston, (1994).

[4] G. Adomian, Solutions of nonlinear P.D.E, Appl. Math. Lett., 11 (1998), 121-123.

[5] Y. Zhu, Q. Chang and S. Wu, A new algorithm for calculating Adomian polynomials, Appl. Math. Comp., 169 (2005), 402-416.

[6] K. Diethelm, The Analysis Fractional Differential Equations, Springer-Verlag Berlin Heidelberg (2010).

[7] I. Podlubny, Fractional Differential Equations, Academic Press, San Diego, CA, (1999).

[8] A. K. H. Sedeeg and M. M. A. Mahgoub, Comparison of New Integral Transform Aboodh Transform and Adomian Decomposition Method, Int. J. Math. And App., 4 (2-B) (2016), 127-135.

[9] D. Kumar, J. Singh and S. Rathore, Sumudu Decomposition Method for Nonlinear Equations, Int. Math. For., 7 (2012), $515-521$.

[10] D. Ziane and M. Hamdi Cherif, Resolution of Nonlinear Partial Differential Equations by Elzaki Transform Decomposition Method, J. Appr. Theor. App. Math., 5 (2015), 17-30.

[11] H. M. Baskonus, H. Bulut and Y. Pandir, The natural transform decomposition method for linear and nonlinear partial differential equations, Math. Engineer. Scie. Aerospace., 5 (1) (2014), 111-126. 
[12] K. S. Aboodh, The new integral transform "Aboodh Transform" , Glob. J. Pure. App. Math., 9 (1) (2013), 35-43.

[13] M. M. A. Mahgoub and A. K. H. Sedeeg, An Efficient Method for Solving Linear and Nonlinear System of Partial Differential Equations, British. J. Math. Comp. Sci., 20 (1) (2017), 1-10.

[14] J. Ahmad, Z. Bibi and K. Noor, Laplace decomposition method using He's polynomial to Burgers equation, J. Scie. Arts., 2 (27) (2014), 131-138.

[15] M. M. A. Mahgoub and A. K. H. Sedeeg, A Comparative Study of Homotopy Perturbation Aboodh Transform Method and Homotopy Decomposition Method for Solving Nonlinear Fractional Partial Differential Equations, Int. J. Theor. App. Math., 2 (2) (2016), 45-51.

[16] R. I. Nuruddeen and K. S. Aboodh, Analytical Solution For Time-Fractional Diffusion Equation By Aboodh Decomposition Method, Int. J. Math. And App., 5 (1-A) (2017), 115-122.

[17] R. I. Nuruddeen and A. M. Nass, Aboodh Decomposition Method and its Application in Solving Linear and Nonlinear Heat Equations, Europ. J. Adv. Eng. Tech., 3 (7) (2016), 34-37.

${ }^{1}$ Department of Mathematics and Informatics, Electrical and Energy Engineering Graduate School (ESGEE), Oran, Algeria., Laboratory of mathematics and its applications (LAMAP), University of Oran1 Ahmed Ben Bella, Oran, Algeria.

${ }^{2}$ Laboratory of mathematics and its applications (LAmAP), University of Oran1 Ahmed Ben Bella, Oran, 31000, Algeria.

*Corresponding AUthor: mountassir27@yahoo.fr 